\title{
BOGOMOLOV MULTIPLIERS OF $p$-GROUPS OF MAXIMAL CLASS
}

\author{
GUSTAVO A. FERNÁNDEZ-ALCOBER AND URBAN JEZERNIK
}

\begin{abstract}
Let $G$ be a $p$-group of maximal class and order $p^{n}$. We determine whether or not the Bogomolov multiplier $\mathrm{B}_{0}(G)$ is trivial in terms of the lower central series of $G$ and $P_{1}=C_{G}\left(\gamma_{2}(G) / \gamma_{4}(G)\right)$. If in addition $G$ has positive degree of commutativity and $P_{1}$ is metabelian, we show how understanding $\mathrm{B}_{0}(G)$ reduces to the simpler commutator structure of $P_{1}$. This result covers all $p$-groups of maximal class of large enough order and, furthermore, it allows us to give the first natural family of $p$-groups containing an abundance of groups with nontrivial Bogomolov multipliers. We also provide more general results on Bogomolov multipliers of $p$-groups of arbitrary coclass $r$.
\end{abstract}

\section{INTRODUCTION}

The Bogomolov multiplier is a group-theoretical invariant, originally introduced as an obstruction to the famous rationality problem in algebraic geometry. This problem asks whether a given field extension $E / k$ is rational (purely transcendental). Of particular interest is the situation when a finite group $G$ acts on the function field $L$ of an affine space by permuting indeterminates. The subfield $L^{G}$ of fixed points of this action represents the function field of the quotient variety. Noether [25] asked whether the extension $L^{G} / k$ is always (stably) purely transcendental. This version of the rationality problem is known as Noether's problem. Saltman [26] found explicit examples of groups for which the answer to Noether's problem is in general negative, even when taking $k=\mathbb{C}$. His approach was to use a certain Galois-cohomological invariant associated to the group $G$, namely, the unramified Brauer group. It was Bogomolov [3] who showed that this invariant can be computed purely in terms of group-cohomology as the intersection of kernels of all restriction maps from the Schur multiplier of $G$ to Schur multipliers of its abelian subgroups:

$$
\bigcap_{A \leq G,[A, A]=1} \operatorname{ker}\left(\mathrm{H}^{2}\left(G, \mathbb{C}^{*}\right) \longrightarrow \mathrm{H}^{2}\left(A, \mathbb{C}^{*}\right)\right) \text {. }
$$

This cohomological invariant is now known as the Bogomolov multiplier and denoted by $\mathrm{B}_{0}(G)$. It represents an obstruction to Noether's problem, which has a negative answer for $G$ provided that $\mathrm{B}_{0}(G)$ is nontrivial.

Explicitly determining the Bogomolov multiplier of a group $G$ can be quite involved. By general homological arguments, it boils down to studying Bogomolov

Date: September 2, 2019.

Key words and phrases. Bogomolov multiplier, $p$-group of maximal class.

This project was supported by the Spanish Government, grant MTM2017-86802-P, partly with FEDER funds, and by the Basque Government, grant IT974-16. This project has also received funding from the European Union's Horizon 2020 research and innovation programme under the Marie Sklodowska-Curie grant agreement No 748129. 
multipliers of the Sylow subgroups of $G$. For $p$ a prime, the smallest examples of $p$-groups with nontrivial Bogomolov multiplier are of order 64 if $p=2[5,4]$, and of order $p^{5}$ if $p$ is odd $[3,23]$. These groups are all of maximum nilpotency class given their order.

Motivated by these examples, in this paper we set to inspect Bogomolov multipliers of $p$-groups $G$ of maximal class, that is, of order $p^{n}$ and nilpotency class $n-1$. Our study of Bogomolov multipliers of these groups partially builds on and continues previous works on Schur multipliers of $p$-groups of maximal class and related groups $[6,8,9,22]$. The theory of $p$-groups of maximal class was started by Blackburn in [2] and further developed by Leedham-Green and McKay [18, 19, 20], by Shepherd [29], and by the first author [12]. General accounts of this theory can be found in [13], [14] or [21]. We can assume $n \geq 4$, and we will tacitly do so in what follows. Consider the chief series $G>P_{1}>\cdots>P_{n}=1$, with $P_{i}=\gamma_{i}(G)$ for $i \geq 2$, and $P_{1}=C_{G}\left(P_{2} / P_{4}\right)$. Then $P_{1}$ is a maximal subgroup of $G$. As a main result, we prove that most $p$-groups of maximal class have non-trivial Bogomolov multipliers.

Theorem 1.1. Let $G$ be a p-group of maximal class and order $p^{n}$. Then $\mathrm{B}_{0}(G)$ is trivial if and only if $\left[P_{1}, P_{1}\right]=\left[P_{1}, P_{n-2}\right]$.

The degree of commutativity of $G$ is the largest integer $\ell \leq n-3$ such that $\left[P_{i}, P_{j}\right] \leq P_{i+j+\ell}$ for all $i, j \geq 1$. If we assume in the above theorem that $G$ has positive degree of commutativity, i.e., $\ell>0$, then we have that $G$ has trivial Bogomolov multiplier precisely when $P_{1}$ is abelian. This assumption on $\ell$ is very natural when dealing with $p$-groups of maximal class, and in fact it is satisfied whenever $n>p+1$ [21, Theorem 3.3.5].

Corollary 1.2. Let $G$ be a p-group of maximal class of positive degree of commutativity. Then $B_{0}(G)$ is trivial if and only if $P_{1}$ is abelian.

We remark that an abelian-by-cyclic finite group is already known to have trivial Bogomolov multiplier by [3, Lemma 4.9]. As a simple example, consider the special cases $p \in\{2,3\}$. When $p=2$, all the groups have abelian $P_{1}$, and therefore trivial Bogomolov multipliers. When $p=3$, all the groups have positive degree of commutativity and $\left|\left[P_{1}, P_{1}\right]\right| \leq 3$, so we either have that $P_{1}$ is abelian, in which case $\mathrm{B}_{0}(G)$ is trivial, or $\left[P_{1}, P_{1}\right]=P_{n-1}$ and the degree is positive, in which case $\mathrm{B}_{0}(G)$ is nontrivial.

Explicitly determining the structure of $\mathrm{B}_{0}(G)$ is rather complicated even in the case when $P_{1}$ is assumed to be metabelian, still assuming that $G$ has positive degree of commutativity. As we will see, both assumptions are satisfied if $n$ is large enough with respect to $p$. Therefore we focus on this case, and we give in Theorem $2.1 \mathrm{a}$ precise description of the Bogomolov multiplier in terms of the curly exterior square introduced by Moravec [22].

Remarkably, this allows us to describe the first natural example of a family of p-groups having Bogomolov multipliers of unbounded exponent (see Section 3). 
Theorem 1.3. Let $p \geq 5$ be a prime, and let $m$ and $n$ be integers such that $m \geq 4$ and $m \leq n \leq 2 m-2$. If we write $n-m+1=x(p-1)+y$ with $x \geq 0$ and $0 \leq y<p-1$, then there exists a p-group of maximal class of order $p^{n}$ with $P_{1}^{\prime}=P_{m}$ and Bogomolov multiplier isomorphic to

$$
C_{p^{x+1}}^{\lfloor y / 2\rfloor} \times C_{p^{x}}^{\lfloor(p-1-y) / 2\rfloor} .
$$

Finally we also explore Bogomolov multipliers of $p$-groups of general coclass $r$, that is, groups of order $p^{n}$ and nilpotency class $n-r$ for some $r \geq 1$. In taking $r=1$, one recovers finite $p$-groups of maximal class. By general coclass theory (see Section 4 ), there is a way of collecting all but finitely many of these groups into a finite number of infinite trees arising from pro- $p$ groups. We investigate the structure of Bogomolov as well as Schur multipliers from this point of view; we improve a result on the asymptotic shape of the Schur multiplier for infinite coclass sequences of groups [9, Theorem 1], and we extend the same result to the Bogomolov multiplier.

\section{MAXIMAL CLASS}

In this section, we prove the general criterion for deciding whether or not Bogomolov multipliers of $p$-groups of maximal class are trivial, Theorem 1.1. This is done by first proving the more technical Theorem 2.1, from which we can deduce one of the implications of Theorem 1.1, and then we complete the proof of this theorem.

Before proceeding, we will collect some well-known structural properties of $p$ groups of maximal class that we will require later [13, 14, 21]. First of all, recall that we have a chief series $G>P_{1}>\cdots>P_{n}=1$ with $P_{i}=\gamma_{i}(G)$ for $i \geq 2$. Observe that $P_{i}=1$ for $i \geq n$, and that $P_{2}, \ldots, P_{n}$ are the only normal subgroups of $G$ of index greater than $p$. If we pick arbitrary elements $s \in G \backslash\left(P_{1} \cup C_{G}\left(P_{n-2}\right)\right)$ and $s_{1} \in P_{1} \backslash P_{2}$, then $s$ and $s_{1}$ generate $G$. We call $s$ a uniformizing element. Also, we have $C_{G}(s)=\langle s\rangle P_{n-1}$, which is of order $p^{2}$. Let us define $s_{i}=\left[s_{i-1}, s\right]$ for all $i \geq 2$. Then $s_{i} \in P_{i} \backslash P_{i+1}$ for $1 \leq i \leq n-1$, and $s_{i}=1$ for $i \geq n$. As a consequence, every $g \in G$ can be uniquely written in the form

$$
g=s^{i_{0}} s_{1}^{i_{1}} \ldots s_{n-1}^{i_{n-1}},
$$

with $0 \leq i_{j}<p$ for all $j=0, \ldots, n-1$. We refer to this as the normal form of $g$ with respect to $s$ and the $s_{i}$. Let $\ell$ be the degree of commutativity of $G$. Then $\ell \geq 0$, and $\ell=n-3$ if and only if $P_{1}$ is abelian. We write $\ell(G)$ when we want to emphasize the group $G$. A fundamental result of Blackburn is that $\ell>0$ if $n \geq p+2$. On the other hand, we have $\ell(G / N) \geq \ell(G)$ for every $N \triangleleft G$. Also, $\ell(G / Z(G))$ is always positive and, as a consequence, $\ell(G)=0$ if and only if $\left[P_{1}, P_{n-2}\right]=P_{n-1}$.

Let us also recall some of the basic facts related to Bogomolov multipliers $\mathrm{B}_{0}(G)$ for arbitrary groups $G$. In this paper, we will work exclusively with a combinatorial interpretation of the Bogomolov multiplier as developed by Moravec [24]. Namely, consider the curly exterior square $G \curlywedge G$, which is the group generated by the symbols $x \curlywedge y$ for all pairs $x, y \in G$, subject to the relations

$$
x y \curlywedge z=\left(x^{y} \curlywedge z^{y}\right)(y \curlywedge z), \quad x \curlywedge y z=(x \curlywedge z)\left(x^{z} \curlywedge y^{z}\right), \quad a \curlywedge b=1,
$$


for all $x, y, z \in G$ and all $a, b \in G$ with $[a, b]=1$. By [24, Theorem 3.2], there is a canonical epimorphism $G \curlywedge G \rightarrow[G, G]$, whose kernel is isomorphic to $\mathrm{B}_{0}(G)$. In this sense, the Bogomolov multiplier can be interpreted as a measure of how the commutator relations in a group fail to follow from the so-called universal ones and from commuting pairs in $G$, see [16]. This can be taken a step further if one has an explicit presentation of the underlying group $G$ as $F / R$ with $F$ a free group. Let $\mathrm{K}(F)=\{[f, g] \mid f, g \in F\}$ denote the set of simple commutators in $F$. Note that $\langle\mathrm{K}(F) \cap R\rangle$ contains $[R, F]$. We then have Hopf-type formulas [24, Proposition 3.8] for both the curly exterior square and the Bogomolov multiplier,

$$
G \curlywedge G \cong \frac{F^{\prime}}{\langle\mathrm{K}(F) \cap R\rangle} \quad \text { and } \quad \mathrm{B}_{0}(G) \cong \frac{F^{\prime} \cap R}{\langle\mathrm{~K}(F) \cap R\rangle} .
$$

Our approach to proving statements about a $p$-group of maximal class $G$ will be to use the above formulae. We also remark that in such a group, all elements of $G^{\prime}$ are simple commutators of the form $[s, g]$ with $g \in G$ and then, by [24, Theorem $3.11]$, we have an epimorphism $\mathrm{B}_{0}(G) \longrightarrow \mathrm{B}_{0}\left(G / P_{i}\right)$ for every $i \geq 2$.

We are now ready to give a precise description of the Bogomolov multiplier of most $p$-groups of maximal class in terms of the curly exterior square of a maximal subgroup.

Theorem 2.1. Let $G$ be a p-group of maximal class with positive degree of commutativity and $P_{1}^{\prime}$ abelian. Let $s \in G \backslash P_{1}$ be a uniformizing element. Then $\mathrm{B}_{0}(G)$ is isomorphic to the coinvariants $\left(P_{1} \curlywedge P_{1}\right)_{\langle s\rangle}$.

Here, $P_{1} \curlywedge P_{1}$ is seen as an $\langle s\rangle$-module under the action induced by the rule $(x \curlywedge y)^{s}=x^{s} \curlywedge y^{s}$ for all $x, y \in P_{1}$.

Proof of Theorem 2.1. It is known that any $p$-group of maximal class $G$ admits a presentation that can be constructed as follows (see [21, Exercise 3.3(4)]). Let $F$ be the free group on $t$ and $t_{1}$. Denote $t_{i}=\left[t_{i-1}, t\right]$ for all $i \geq 2$. Every element $g$ of $G$ has a normal form in terms of the generating set $s$ and $s_{i}$ for $1 \leq i \leq n-1$. For a word $w$ of $F$, let $\llbracket w \rrbracket$ denote the word in $t$ and $t_{i}$ for $1 \leq i \leq n-1$ obtained by replacing $s$ with $t$ and $s_{i}$ with $t_{i}$ in the normal form of the element of $G$ that is represented by the word $w$. Denote by $\rho(w)=w \llbracket w \rrbracket^{-1}$ the relator associated to $w$. Set

$$
\mathcal{R}_{0}=\left\{t_{n}\right\}, \mathcal{R}_{1}=\left\{\rho\left(t^{p}\right), \rho\left(\left(t t_{1}\right)^{p}\right)\right\}, \mathcal{R}_{2}=\left\{\rho\left(\left[s_{2 i}, s_{1}\right]\right) \mid 1 \leq i \leq(p-1) / 2\right\},
$$

and let $R$ be the normal subgroup of $F$ generated by $\mathcal{R}_{0} \cup \mathcal{R}_{1} \cup \mathcal{R}_{2}$. Then $F / R$ is a presentation of the group $G$. We denote by $M=\left\langle t_{1}\right\rangle^{F} R$ the maximal subgroup of $F$ that maps onto $P_{1}$, and we consider the map

$$
\lambda: M^{\prime} \longrightarrow \frac{F^{\prime} \cap R}{\langle\mathrm{~K}(F) \cap R\rangle} \cong \mathrm{B}_{0}(G), \quad w \longmapsto \rho(w)\langle\mathrm{K}(F) \cap R\rangle .
$$

Observe that $\left(P_{1} \curlywedge P_{1}\right)_{\langle s\rangle}$ is isomorphic to the group $M^{\prime} /\left(\langle\mathrm{K}(M) \cap R\rangle\left[M^{\prime}, t\right]\right)$. Our objective is to prove that $\lambda$ induces an isomorphism

$$
\bar{\lambda}: \frac{M^{\prime}}{\langle\mathrm{K}(M) \cap R\rangle\left[M^{\prime}, t\right]} \cong\left(P_{1} \curlywedge P_{1}\right)_{\langle s\rangle} \longrightarrow \frac{F^{\prime} \cap R}{\langle\mathrm{~K}(F) \cap R\rangle} \cong \mathrm{B}_{0}(G) .
$$


I. We show that $\left\langle t_{m}, \ldots, t_{n-1}\right\rangle \cap R \leq\langle\mathrm{K}(F) \cap R\rangle$, where $m$ is the smallest index with $\left[P_{1}, P_{1}\right]=P_{m}$. To this end, let $\omega \in\left\langle t_{m}, \ldots, t_{n-1}\right\rangle \cap R$. Observe that since $P_{m}$ is assumed to be abelian, we have $\left[\gamma_{m}(F), \gamma_{m}(F)\right] \leq\langle\mathrm{K}(F) \cap R\rangle$, and so $\omega$ can be written as

$$
\omega \equiv \prod_{i=m}^{n-1} t_{i}^{a_{i}} \quad(\bmod \langle\mathrm{K}(F) \cap R\rangle)
$$

with $a_{m} \equiv 0(\bmod p)$. Note that

$$
\left[t_{m-1}^{p}, t\right]=t_{m}^{t_{m-1}^{p-1}} t_{m}^{t_{m-1}^{p-2}} \cdots t_{m} \equiv t_{m}^{p}\left[t_{m}, t_{m-1}^{\left(\begin{array}{c}
p \\
2
\end{array}\right)}\right] \quad(\bmod \langle\mathrm{K}(F) \cap R\rangle) .
$$

Now, if $p>2$, then $t_{m-1}^{\left(\begin{array}{c}p \\ 2\end{array}\right)} \in P_{m}$, and in the case when $p=2, P_{m-1}=P_{n-1}$ is central in $G$. We therefore always have $\left[t_{m}, t_{m-1}^{\left(\begin{array}{c}p \\ 2\end{array}\right)}\right] \in \mathrm{K}(F) \cap R$. It follows that

$$
\omega \equiv\left[\prod_{i=m}^{n-1} t_{i-1}^{a_{i}}, t\right] \quad(\bmod \langle\mathrm{K}(F) \cap R\rangle),
$$

and so $\omega \in\langle\mathrm{K}(F) \cap R\rangle$, as desired.

II. We claim that $\lambda$ is a homomorphism. To see this, first observe that since $P_{m}$ is assumed to be abelian, we have $\left[M^{\prime} \gamma_{m}(F), M^{\prime} \gamma_{m}(F)\right] \leq R$. Now pick any $x, y \in M^{\prime}$. Note that $\llbracket x \rrbracket, \llbracket y \rrbracket \in \gamma_{m}(F)$. Hence

$$
\lambda(x) \lambda(y)=x \llbracket x \rrbracket^{-1} y \llbracket y \rrbracket^{-1} \equiv x y(\llbracket x \rrbracket \llbracket y \rrbracket)^{-1} \quad(\bmod \langle\mathrm{K}(F) \cap R\rangle) .
$$

We have $\llbracket x \rrbracket \llbracket y \rrbracket \llbracket x y \rrbracket^{-1} \in R \cap\left\langle t_{m}, \ldots, t_{n-1}\right\rangle \leq\langle\mathrm{K}(F) \cap R\rangle$. We thus conclude

$$
\lambda(x) \lambda(y)=x y \llbracket x y \rrbracket^{-1}=\lambda(x y) \quad(\bmod \langle\mathrm{K}(F) \cap R\rangle) .
$$

III. Let us now show that $\lambda$ is surjective. Consider the group $F /\langle R \cap \mathrm{K}(F)\rangle$. Its subgroup $R /\langle R \cap \mathrm{K}(F)\rangle$ is an abelian group that can be generated by the cosets of the elements of $\mathcal{R}_{1} \cup \mathcal{R}_{2}$. Observe that $R /\left(R \cap F^{\prime}\right) \cong R F^{\prime} / F^{\prime}$ can be generated by the cosets of elements of $\mathcal{R}_{1}$. Moreover, the elements $t^{p} F^{\prime}$ and $t_{1}^{p} F^{\prime}$ form a base of the free abelian group $R F^{\prime} / F^{\prime}$ of rank 2 . Hence we have that the torsion group $\left(R \cap F^{\prime}\right) /\langle R \cap \mathrm{K}(F)\rangle$ is generated by the cosets of the elements of $\mathcal{R}_{2}$. Now note that $\mathcal{R}_{2} \subseteq \rho\left(M^{\prime}\right)$. Therefore $\lambda$ is indeed surjective.

IV. The homomorphism $\lambda$ factors through $\langle\mathrm{K}(M) \cap R\rangle\left[M^{\prime}, t\right]$. It is clear that $\langle\mathrm{K}(M) \cap R\rangle$ is contained in the kernel of $\lambda$. To see that the same holds for $\left[M^{\prime}, t\right]$, consider $\lambda\left(m^{t}\right)$ for some $m \in M^{\prime}$. We have that $\llbracket m^{t} \rrbracket \equiv \llbracket m \rrbracket^{t}$ modulo $\left(R \cap\left\langle t_{m}, \ldots, t_{n-1}\right\rangle\right)\left\langle t_{n}\right\rangle^{F} \leq\langle\mathrm{K}(F) \cap R\rangle$. Whence

$\lambda\left(m^{t}\right)=m^{t} \llbracket m^{t} \rrbracket^{-1} \equiv m^{t} \llbracket m \rrbracket^{-t}=m \llbracket m \rrbracket^{-1}\left[m \llbracket m \rrbracket^{-1}, t\right] \equiv \lambda(m) \quad(\bmod \langle\operatorname{K}(F) \cap R\rangle)$, proving our claim. There is thus an induced homomorphism

$$
\bar{\lambda}: \frac{M^{\prime}}{\langle\mathrm{K}(M) \cap R\rangle\left[M^{\prime}, t\right]} \cong\left(P_{1} \curlywedge P_{1}\right)_{\langle s\rangle} \longrightarrow \frac{F^{\prime} \cap R}{\langle\mathrm{~K}(F) \cap R\rangle} \cong \mathrm{B}_{0}(G) .
$$

V. The homomorphism $\bar{\lambda}$ is injective. To this end, let $w \in M^{\prime}$ represent an element in $\operatorname{ker} \bar{\lambda}$. So $w \llbracket w \rrbracket^{-1} \in\langle\mathrm{K}(F) \cap R\rangle$. Write $w=\llbracket w \rrbracket \cdot \prod_{i}\left[x_{i}, y_{i}\right]$ for some $\left[x_{i}, y_{i}\right] \in R$. Collect all those indices $i$ for which $x_{i}, y_{i} \in M$ into a set $I$. Eventually replacing $w$ by $w \prod_{i \in I}\left[x_{i}, y_{i}\right]^{-1}$, we may assume that $x_{i} \notin M$ and $y_{i} \in M$ for all indices $i$. Write $x_{i}=t^{a_{i}} m_{i}$ for some $1 \leq a_{i}<p$ and $m_{i} \in M$. Since $\left[x_{i}, y_{i}\right] \in$ $R$, it follows that $y_{i}=t_{n-1}^{b_{i}} r_{i}$ for some $0 \leq b_{i}<p$ and $r_{i} \in R$. So $\left[x_{i}, y_{i}\right]=$ 
$\left[t^{a_{i}} m_{i}, t_{n-1}^{b_{i}} r_{i}\right] \equiv\left[t^{a_{i}}, t_{n-1}^{b_{i}} r_{i}\right]\left[m_{i}, t_{n-1}^{b_{i}} r_{i}\right]$. Now $\left[m_{i}, t_{n-1}^{b_{i}} r_{i}\right] \in\langle\mathrm{K}(M) \cap R\rangle$, so that $\left[x_{i}, y_{i}\right] \equiv\left[t, t_{n-1}\right]^{a_{i} b_{i}}\left[t, r_{i}\right]^{a_{i}}$. Thus we may write $w \equiv \llbracket w \rrbracket \cdot\left[t, t_{n-1}^{a}\right][t, r]$ for some $0 \leq a<p$ and $r \in R$. As $R \leq\left\langle M^{\prime}, t^{p}, t_{m}, \ldots, t_{n-1}\right\rangle^{F}$, it follows that $R$ can be generated modulo $R \cap M^{\prime}$ by words in $t^{p}, t_{m}, \ldots, t_{n-1}$. We are in a setting where $\left[M^{\prime}, t\right] \equiv 1$, so we can write $w \equiv \prod_{i \geq m} t_{i}^{c_{i}}$ for some integers $c_{i}$.

Note that since $P_{m}$ is assumed to be abelian, the image of the group $\left\langle t_{i} \mid i \geq m\right\rangle$ in $M /\langle\mathrm{K}(M) \cap R\rangle$ is abelian, and so it is a quotient of the free abelian group generated by the elements $t_{i}$ for $i \geq m$. Moreover, the group $M / M^{\prime}$ is the quotient of the free abelian group generated by the elements $t^{p}$ and $t_{i}$ for $i \geq 1$ subject only to the relations

$$
\prod_{i=1}^{p} t_{j+i}^{\left(\begin{array}{c}
p \\
i
\end{array}\right)} \equiv 1 \quad\left(\bmod M^{\prime}\right)
$$

for all $j \geq 1$. These arise from expanding $\left[t_{j}, t^{p}\right] \in M^{\prime}$. The element $w$ belongs to $M^{\prime}$, therefore it can be written modulo $\langle\mathrm{K}(M) \cap R\rangle$ as a product of some powers of elements of the form $\prod_{i=1}^{p} t_{j+i}^{\left(\begin{array}{c}p \\ i\end{array}\right)}$ for some $j \geq m$. Now, observe that

$$
\prod_{i=1}^{p} t_{j+i}^{\left(\begin{array}{l}
p \\
i
\end{array}\right)} \equiv\left[t_{j}, t^{p}\right] \equiv 1 \quad(\bmod \langle\mathrm{K}(M) \cap R\rangle)
$$

for all $j \geq m-1$. Therefore $w \equiv 1$ in the domain of $\bar{\lambda}$ and the proof is complete.

In the next corollary, we use arithmetical conditions to have high degree of commutativity from [12] in order to show that Theorem 2.1 covers all but finitely many $p$-groups of maximal class for every prime $p$.

Corollary 2.2. Let $G$ be a p-group of maximal class of order $p^{n}$. If

$$
n \geq \max \{p+2,6 p-29\},
$$

then $\mathrm{B}_{0}(G) \cong\left(P_{1} \curlywedge P_{1}\right)_{\langle s\rangle}$.

Proof. The condition $n \geq p+2$ ensures that $G$ has positive degree of commutativity. Hence, according to Theorem 2.1 it suffices to check that $P_{1}$ is metabelian. If $p=2$, 3 or 5 , then the degree of commutativity $\ell$ of $G$ is at least $n-3, n-4$ or $(n-6) / 2$, respectively, and the result readily follows. On the other hand, if $p \geq 7$ then $2 \ell \geq n-2 p+5$ by [12]. Since $P_{1}^{\prime}=\left[P_{1}, P_{2}\right] \leq P_{\ell+3}$, we have

$$
\left[P_{1}^{\prime}, P_{1}^{\prime}\right] \leq\left[P_{\ell+3}, P_{\ell+3}\right]=\left[P_{\ell+3}, P_{\ell+4}\right] \leq P_{3 \ell+7},
$$

where

$$
3 \ell+7 \geq \frac{3}{2}(n-2 p+5)+7=n+\frac{1}{2}(n-6 p+29) \geq n,
$$

since $n \geq 6 p-29$ by hypothesis. Thus $P_{1}$ is metabelian, as desired.

Now we continue by proving Theorem 1.1. We will use Theorem 2.1 for this together with the fact that, if $\left[P_{1}, P_{1}\right]=P_{m}$, then $\mathrm{B}_{0}(P)$ maps onto $\mathrm{B}_{0}\left(P / P_{m+1}\right)$.

Proof of Theorem 1.1. We first assume that $\left[P_{1}, P_{1}\right]=P_{m}$ is strictly larger than $\left[P_{1}, P_{n-2}\right]$, and prove that $\mathrm{B}_{0}(G)$ is nontrivial. If $\ell(G)=0$ then $\left[P_{1}, P_{n-2}\right]=P_{n-1}$, and so $G / P_{m+1}$ is a proper quotient of $G$. We conclude that $\ell\left(G / P_{m+1}\right)>0$ in 
every case. Then we may apply Theorem 2.1 to $G / P_{m+1}$ to get $\mathrm{B}_{0}\left(G / P_{m+1}\right) \cong$ $\left(P_{1} / P_{m+1} \curlywedge P_{1} / P_{m+1}\right)_{G}$. Since $\mathrm{B}_{0}(G)$ surjects onto $\mathrm{B}_{0}\left(G / P_{m+1}\right)$ and $\left(P_{1} / P_{m+1} \curlywedge\right.$ $\left.P_{1} / P_{m+1}\right)_{G}$ surjects onto $\left[P_{1} / P_{m+1}, P_{1} / P_{m+1}\right]_{G}=\left(P_{m} / P_{m+1}\right)_{G} \cong C_{p}$, we conclude that $\mathrm{B}_{0}(G) \neq 1$.

Now we prove the converse, namely that the condition $\left[P_{1}, P_{1}\right]=\left[P_{1}, P_{n-2}\right]$ implies that $\mathrm{B}_{0}(G)=1$. If $P_{1}$ is abelian, then $G$ is abelian-by-cyclic and hence $\mathrm{B}_{0}(G)$ is trivial by [3]. So assume that $P_{1}$ is not abelian. The restriction $\left[P_{1}, P_{1}\right]=$ $\left[P_{1}, P_{n-2}\right]$ gives $\left[P_{1}, P_{1}\right]=P_{n-1}$. Note that $P_{n-1}$ is generated by the element $s_{n-1}$ of order $p$. Moreover, since $\left[P_{1}, P_{n-2}\right]=P_{n-1}$ and $\left[P_{2}, P_{n-2}\right]=1$, there exists a $\lambda \neq 0 \bmod p$ with $\left[s_{1}, s_{n-2}\right]=s_{n-1}^{\lambda}$. The latter equality may be rewritten as $\left[s^{\lambda} s_{1}, s_{n-2}\right]=1$. Expanding $1=s^{\lambda} s_{1} \curlywedge s_{n-2}$ in the curly exterior square $G \curlywedge G$ gives

$\left(s^{\lambda} \curlywedge s_{n-2}\right)^{s_{1}}\left(s_{1} \curlywedge s_{n-2}\right)=\left(s^{\lambda}\left[s^{\lambda}, s_{1}\right] \curlywedge s_{n-2} s_{n-1}^{-\lambda}\right)\left(s_{1} \curlywedge s_{n-2}\right)=\left(s \curlywedge s_{n-2}\right)^{\lambda}\left(s_{1} \curlywedge s_{n-2}\right)$, therefore $\left(s_{n-2} \curlywedge s\right)^{\lambda}=\left(s_{1} \curlywedge s_{n-2}\right)$. Furthermore, pick any $s_{i}, s_{j}, s_{k}, s_{l}$ in $P_{1}$ and assume that both of the elementary wedges $s_{i} \curlywedge s_{j}$ and $s_{k} \curlywedge s_{l}$ are nontrivial in $G \curlywedge G$. As $\left[P_{1}, P_{1}\right]=P_{n-1}$, both of the commutators $\left[s_{i}, s_{j}\right]$ and $\left[s_{k}, s_{l}\right]$ equal a power of $s_{n-1}$. Since $\mathrm{B}_{0}\left(P_{1}\right)$ is trivial by [16, Corollary 4.1], there exists an $m>0$ such that $\left(s_{i} \curlywedge s_{j}\right)\left(s_{k} \curlywedge s_{l}\right)^{m} \in \mathrm{B}_{0}\left(P_{1}\right)$ is trivial. The natural homomorphism $\mathrm{B}_{0}\left(P_{1}\right) \rightarrow \mathrm{B}_{0}(G)$ shows that $\left(s_{i} \curlywedge s_{j}\right)\left(s_{k} \curlywedge s_{l}\right)^{m}$ is also trivial in $G \curlywedge G$. Hence all the elementary wedges $s_{i} \curlywedge s_{j}$ are equal to a power of the nontrivial one $s_{1} \curlywedge s_{n-2}$.

Now let $w$ be an arbitrary element of $\mathrm{B}_{0}(G)$. For any $x, y \in P_{1}$ and $g, h \in G$, we have $[x \curlywedge y, g \curlywedge h]=[x, y] \curlywedge[g, h]=1$ in $G \curlywedge G$, since $P_{n-1}=Z(G)$. Note also that $\left[s_{i} \curlywedge s, s_{j} \curlywedge s\right]=s_{i+1} \curlywedge s_{j+1}$. Therefore the element $w$ can be written as

$$
w=\prod_{i=1}^{n-2}\left(s_{i} \curlywedge s\right)^{\alpha_{i}} \cdot\left(s_{1} \curlywedge s_{n-2}\right)^{\beta}
$$

for some integers $\alpha_{i}, \beta$. Observe that

$$
1=s_{i} \curlywedge s^{p}=\left(s_{i} \curlywedge s^{p-1}\right)\left(s_{i} \curlywedge s\right)^{s^{p-1}}=\left(s_{i} \curlywedge s\right)^{p} \cdot \prod_{j>i}\left(s_{j} \curlywedge s\right)^{a_{j}} \cdot\left(s_{1} \curlywedge s_{n-2}\right)^{b}
$$

for some $a_{j}, b$. We may thus assume that $0 \leq \alpha_{i}<p$, and the same for $\beta$. Note that $w$ belongs to $\mathrm{B}_{0}(G)$ if and only if we have $\prod_{i=1}^{n-2} s_{i+1}^{\alpha_{i}} \cdot\left[s_{1}, s_{n-2}\right]^{\beta}=1$ in $G$. Collecting the left hand side in its normal form and comparing exponents gives $\alpha_{i}=0$ for all $i \leq n-3$ and $\alpha_{n-2}+\lambda \beta=0$. We thus have $w=\left(\left(s \curlywedge s_{n-2}\right)^{\lambda}\left(s_{1} \curlywedge s_{n-2}\right)\right)^{\beta}$, and so $w=1$ by above. Hence $\mathrm{B}_{0}(G)$ is trivial, as required.

\section{EXAMPles}

In this section, we show how Theorem 2.1 can be utilized to explicitly determine the structure of Bogomolov multipliers of some particular $p$-groups of maximal class (for $p \geq 5$ ). As a consequence, we prove Theorem 1.3.

To do this, we recall that the structure of a $p$-group $G$ of maximal class with $P_{1}$ of nilpotency class 2 can be given in terms of the ring of integers in the $p$-th cyclotomic number field $\mathcal{O}$. So $\mathcal{O}=\mathbb{Z}[\theta] /\left(1+\theta+\cdots+\theta^{p-1}\right)$, where $\theta$ is a primitive complex $p$-th root of unity. Denote $\kappa=\theta-1$ and let $\mathfrak{p}=(\kappa)$. There is an action 
of $\mathcal{O}$ on $P_{m}$ with $\theta$ acting via conjugation by $s$. By [21, Lemma 8.2.1], there is an $\mathcal{O}$-module isomorphism between $P_{i} / P_{i+j}$ and $\mathcal{O} / \mathfrak{p}^{j}$, induced by the map

$$
\mathcal{O} \rightarrow P_{i} / P_{i+j}, \quad \sum_{u} a_{u} \kappa^{u} \mapsto \prod_{u} s_{i+u}^{a_{u}} .
$$

The commutator structure of $P_{1}$ can thus be understood in terms of the homomorphism

$$
\alpha: \mathcal{O} / \mathfrak{p}^{m-1} \wedge \mathcal{O} / \mathfrak{p}^{m-1} \rightarrow \mathcal{O} / \mathfrak{p}^{n-m} \cong P_{m}
$$

This is in fact a homomorphism of $\langle\theta\rangle=C_{p}$ modules. Set

$$
\mathrm{K} \alpha=\left\langle\operatorname{ker} \alpha \cap\left\{\text { elementary wedges in } \mathcal{O} / \mathfrak{p}^{m-1} \wedge \mathcal{O} / \mathfrak{p}^{m-1}\right\}\right\rangle .
$$

Now consider the induced epimorphism

$$
\alpha_{C_{p}}:\left(\mathcal{O} / \mathfrak{p}^{m-1} \wedge \mathcal{O} / \mathfrak{p}^{m-1}\right)_{C_{p}} \rightarrow\left(\mathcal{O} / \mathfrak{p}^{n-m}\right)_{C_{p}} \cong \mathcal{O} / \mathfrak{p} \cong P_{m} / P_{m+1} \cong C_{p}
$$

obtained by factoring out the action of $\theta$. Correspondingly, there is the induced kernel

$$
\mathrm{K} \alpha_{C_{p}}=\left\langle\operatorname{ker} \alpha_{C_{p}} \cap\left\{\text { image of elementary wedges in }\left(\mathcal{O} / \mathfrak{p}^{m-1} \wedge \mathcal{O} / \mathfrak{p}^{m-1}\right)_{C_{p}}\right\}\right\rangle .
$$

Notice that

$$
\frac{\left(\mathcal{O} / \mathfrak{p}^{m-1} \wedge \mathcal{O} / \mathfrak{p}^{m-1}\right)_{C_{p}}}{\mathrm{~K} \alpha_{C_{p}}} \cong\left(\frac{\mathcal{O} / \mathfrak{p}^{m-1} \wedge \mathcal{O} / \mathfrak{p}^{m-1}}{\mathrm{~K} \alpha}\right)_{C_{p}}
$$

by right-exactness of coinvariants. We make the following identification:

$$
P_{1} \curlywedge P_{1}=\frac{P_{1} / P_{m} \wedge P_{1} / P_{m}}{\left\langle x P_{m} \wedge y P_{m} \mid[x, y]=1\right\rangle} \cong \frac{\mathcal{O} / \mathfrak{p}^{m-1} \wedge \mathcal{O} / \mathfrak{p}^{m-1}}{\mathrm{~K} \alpha}
$$

Now, to provide concrete examples, we show that by carefully selecting the map $\alpha$, which in turn determines the group $G$, one may achieve that the image of the map $\mathrm{K} \alpha_{C_{p}}$ in $\left(\mathcal{O} / \mathfrak{p}^{m-1} \wedge \mathcal{O} / \mathfrak{p}^{m-1}\right)_{C_{p}}$ is trivial. Based on the above identification, this amounts to constructing groups $G$ with $\mathrm{B}_{0}(G) \cong\left(\mathcal{O} / \mathfrak{p}^{m-1} \wedge \mathcal{O} / \mathfrak{p}^{m-1}\right)_{C_{p}}$. Such a commutator structure will therefore produce groups whose Bogomolov multipliers will have largest possible rank and exponent for the given values of $n$ and $m$. Furthermore, essentially the same argument will deal with quotients of such extreme groups. The construction we give below covers this more general case.

Fix any $m \geq 4$ and set $\ell=m-3$. The number $\ell$ will be the degree of commutativity of the constructed group. Now pick any $n$ satisfying $m<n \leq 2 m-2$. Set $\mu=n-m+2$, so that $2<\mu \leq m$. Let $g$ be a primitive root modulo $p$ and pick an integer $a$ so that $a \equiv(g+1)^{-1}(\bmod p)$. It is here that we need $p \geq 5$. In the case when $a>(p-1) / 2$, replace $a$ by $1-a$, so that in the end, $2 \leq a \leq(p-1) / 2$. Now define $\alpha: \mathcal{O} / \mathfrak{p}^{m-1} \wedge \mathcal{O} / \mathfrak{p}^{m-1} \rightarrow \mathcal{O} / \mathfrak{p}^{n-m}$ by the rule

$$
\alpha(x \wedge y)=\kappa^{-1} \cdot\left(\sigma_{a}(x) \sigma_{1-a}(y)-\sigma_{a}(y) \sigma_{1-a}(x)\right)
$$

for $x, y \in \mathcal{O} / \mathfrak{p}^{m-1}$. Here, $\sigma_{a}$ is the automorphism of $\mathcal{O} / \mathfrak{p}^{m-1}$ which maps $\theta$ to $\theta^{a}$. This corresponds to the map induced by $\kappa^{-1} S_{a}$ in [21, Theorem 8.3.1]. Set $u_{a}=\left(\theta^{a}-1\right) / \kappa \in \mathcal{O}^{*}$. Then

$$
\alpha\left(\kappa^{i} \wedge \kappa^{j}\right)=\operatorname{sgn}(i-j) \kappa^{i+j-1}\left(u_{a} u_{1-a}\right)^{\min \{i, j\}}\left(u_{a}^{|i-j|}-u_{1-a}^{|i-j|}\right) \in \mathfrak{p}^{i+j-1} .
$$


Observe that $u_{a}^{|i-j|}-u_{1-a}^{|i-j|} \equiv a^{|i-j|}-(1-a)^{|i-j|}(\bmod \mathfrak{p})$. This element belongs to $\mathfrak{p}$ if and only if we have $\left(a^{-1}-1\right)^{|i-j|} \equiv 1(\bmod p)$. By our choice of $a$, this occurs precisely when $i \equiv j(\bmod p-1)$. The commutator map $\alpha$ therefore satisfies $\alpha\left(\kappa^{i} \wedge \kappa^{j}\right) \in \mathfrak{p}^{i+j-1} \backslash \mathfrak{p}^{i+j}$ whenever $i \not \equiv j(\bmod p-1)$.

Invoking [21, Theorem 8.2.7], there is a $p$-group $G$ of maximal class of order $p^{n}$ whose commutator structure is described by the map $\alpha$ given above. In terms of the $P_{i}$-series of $G$, the above discussion shows that we have $\left[P_{i}, P_{j}\right]=P_{i+j+\ell}$ for all $i, j \geq 1$ that satisfy $i \not \equiv j(\bmod p-1)$.

This highly restricted commutator structure enables us to completely understand commuting pairs of $G$.

Lemma 3.1. Let $x \in P_{i} \backslash P_{i+1}$. Then $C_{P_{i}}(x)=\left\langle x, P_{i+j}\right\rangle$, where $j=\max \{n-2 i-$ $\ell, 1\}$.

Proof. Clearly the right hand side centralizes $x$. Conversely, suppose that $y \in$ $P_{k} \backslash P_{k+1}$ for some $k>i$ and $[x, y]=1$. Assume that $y \notin\langle x\rangle$. If $k \equiv i(\bmod p-1)$, then $y=x^{r} z$ for some $r>0$ and $z \in P_{k^{\prime}} \backslash P_{k^{\prime}+1}$ with $[z, x]=1$ and $k^{\prime} \not \equiv i$ $(\bmod p-1)$. In this case, replace $y$ by $z$ and $k$ by $k^{\prime}$, so that we may assume $k \not \equiv i$ $(\bmod p-1)$. Now, since $\left[P_{i}, P_{k}\right]=P_{i+k+\ell}$ and $\left[P_{i+1}, P_{k}\right]\left[P_{i}, P_{k+1}\right] \leq P_{i+k+\ell+1}$, it follows that $P_{i+k+\ell}=P_{i+k+\ell+1}$, which is only possible when $i+k+\ell \geq n$.

In particular, note that $Z\left(P_{1}\right) \geq P_{\mu} \geq P_{m}$ in the group $G$. Transferring to the $C_{p}$-module $\mathcal{O} / \mathfrak{p}^{m-1} \wedge \mathcal{O} / \mathfrak{p}^{m-1}$, we thus have that the elementary wedges in $\mathfrak{p}^{\mu-1} / \mathfrak{p}^{m-1} \wedge \mathcal{O} / \mathfrak{p}^{m-1}$ are all contained in $\mathrm{K} \alpha$. Using Lemma 3.1 more precisely, we now show that wedges that arise from commuting pairs are, modulo the action of $C_{p}$, nothing but the latter.

Lemma 3.2. $\mathrm{K} \alpha_{C_{p}}=\left(\mathcal{O} / \mathfrak{p}^{m-1} \wedge \mathfrak{p}^{\mu-1} / \mathfrak{p}^{m-1}\right)+\left[\mathcal{O} / \mathfrak{p}^{m-1} \wedge \mathcal{O} / \mathfrak{p}^{m-1}, C_{p}\right]$.

Proof. Let $x \wedge y \in \mathrm{K} \alpha$ for some $x, y \in \mathcal{O} / \mathfrak{p}^{m-1}$. Suppose that $x$ corresponds to an element in $P_{i} \backslash P_{i+1}$ and $y$ to an element in $P_{j} \backslash P_{j+1}$ with $i \leq j$. We will prove that $x \wedge y$ is equivalent to an element of the submodule $\mathfrak{p}^{\mu-1} / \mathfrak{p}^{m-1} \wedge \mathcal{O} / p^{m-1}$ modulo $\left[\mathcal{O} / \mathfrak{p}^{m-1} \wedge \mathcal{O} / \mathfrak{p}^{m-1}, C_{p}\right]$ by induction on $i$.

If $i \equiv j(\bmod p-1)$, then as in the proof of Lemma 3.1, we may write $y=x^{r} z$ with $z \in P_{j^{\prime}} \backslash P_{j^{\prime}+1}$ and $j^{\prime} \not \equiv i(\bmod p-1)$. Then $x \wedge y=x \wedge z$, so we may without loss of generality assume that $i \not \equiv j(\bmod p-1)$. By the lemma, we then have $i+j+\ell \geq n$. If $i=1$, this implies that $j \geq n-\ell-1=\mu$, whence $x \wedge y \in \mathcal{O} / \mathfrak{p}^{m-1} \wedge \mathfrak{p}^{\mu-1} / \mathfrak{p}^{m-1}$. This is the base for the induction. Suppose now that $i>1$. Then $x=\kappa \tilde{x}$ for some $\tilde{x} \in \mathcal{O} / \mathfrak{p}^{m-1}$ corresponding to a group element in $P_{i-1} \backslash P_{i}$. Observe that

$$
\tilde{x} \wedge \kappa y+x \wedge y+x \wedge \kappa y=\kappa(\tilde{x} \wedge y) \in\left[\mathcal{O} / \mathfrak{p}^{m-1} \wedge \mathcal{O} / \mathfrak{p}^{m-1}, C_{p}\right]
$$

Note that $\kappa y$ corresponds to a group element in $P_{j+1}$, and therefore $\tilde{x} \wedge \kappa y$ and $x \wedge \kappa y$ both belong to $\mathrm{K} \alpha$. Using this reasoning, we show our claim by reverse induction on $j$. When $j \geq \mu$, it is clear that $x \wedge y \in \mathcal{O} / \mathfrak{p}^{m-1} \wedge \mathfrak{p}^{\mu-1} / \mathfrak{p}^{m-1}$. Assume 
now that $j<\mu$. By induction, both $\tilde{x} \wedge \kappa y$ (since $\tilde{x}$ belong to a higher level) and $x \wedge \kappa y$ (since $\kappa y$ belongs to a lower level) are contained in $\mathcal{O} / \mathfrak{p}^{m-1} \wedge \mathfrak{p}^{\mu-1} / \mathfrak{p}^{m-1}$ modulo $\left[\mathcal{O} / \mathfrak{p}^{m-1} \wedge \mathcal{O} / \mathfrak{p}^{m-1}, C_{p}\right]$. An application of (3) then implies that the same holds for $x \wedge y$, as claimed.

The above gives that

$$
\mathrm{B}_{0}(G)=\frac{\left(\mathcal{O} / \mathfrak{p}^{m-1} \wedge \mathcal{O} / \mathfrak{p}^{m-1}\right)_{C_{p}}}{\mathrm{~K} \alpha_{C_{P}}}=\left(\mathcal{O} / \mathfrak{p}^{\mu-1} \wedge \mathcal{O} / \mathfrak{p}^{\mu-1}\right)_{C_{p}}
$$

Finally, a structure description of the group $\left(\mathcal{O} / \mathfrak{p}^{\mu-1} \wedge \mathcal{O} / \mathfrak{p}^{\mu-1}\right)_{C_{p}}$ may be read off from the explicit $C_{p}$-module decomposition of $\mathcal{O} / \mathfrak{p}^{\mu-1} \wedge \mathcal{O} / \mathfrak{p}^{\mu-1}$ into a direct sum of cyclic submodules as given in [20, Theorem 8.13]. Then Theorem 1.3 readily follows.

Let us consider some special cases of Theorem 1.3. When $n$ is chosen so that $n \equiv m-1(\bmod p-1)$, we obtain a group $G$ with $\mathrm{B}_{0}(G)$ homocyclic of rank $(p-1) / 2$ and exponent $p^{(n-m+1) /(p-1)}$. Further selecting $n \approx 2 m$, we have the property $\exp \mathrm{B}_{0}(G) \approx \sqrt{\exp G}$. Consider now the option $n=m+1$. In this case, we obtain groups that are immediate descendants of groups on the main line of the maximal class tree. Their Bogomolov multipliers are $C_{p}$. In the very special case when $m=4$, we obtain the known groups of order $p^{5}$ with nontrivial Bogomolov multipliers. Another extreme option is picking $n=2 m-2$. In this case, we have $n-m+1=m-1$, so by varying $m$, the groups exhaust all the possibilities for the Bogomolov multiplier, depending on the value $m-1(\bmod p-1)$. Finally, consider the option of selecting consecutive values $n=m+1, m+2, \ldots, 2 m-2$. In terms of the constructed groups, this corresponds to a path in the maximal class tree, starting from an immediate descendant of a group on the main line (which is of order $p^{m}$ ) and going deeper into the branch. In this process, the value $n-m+1$ grows one by one, so that the corresponding Bogomolov multipliers grow in size by $p$ on each second step, starting with $C_{p}$ for the group closest to the main line. The growth is "staircase"-like, consecutively increasing the orders of the generators by a factor of $p$ on each second step.

The above value is in fact the largest possible for the rank of the Bogomolov multiplier of any $p$-group of maximal class, as the following result shows.

Corollary 3.3. Let $G$ be a p-group of maximal class. Then $\operatorname{rank} \mathrm{B}_{0}(G) \leq(p-1) / 2$.

Proof. In analogy to the Schur multiplier, given a presentation of any finite group $G$ consisting of $d$ generators and $r$ relations among which $r_{k}$ are commutators, the rank of $\mathrm{B}_{0}(G)$ is bounded by $r-r_{k}-d$ (see [17, Corollary 5.2]). Now, we recalled at the beginning of the proof of Theorem 2.1 that any $p$-group of maximal class admits a presentation with $d=2, r=(p+5) / 2$ and $r_{k}=1$, and this proves the result. 
The above exponents are also the largest possible for groups in which $P_{1}$ is of nilpotency class 2 . This is so because if $\left[P_{1}, P_{1}\right]=P_{m}$, then $\mathrm{B}_{0}(G)$ is a quotient of the group $\left(\mathcal{O} / \mathfrak{p}^{m-1} \wedge \mathcal{O} / \mathfrak{p}^{m-1}\right)_{C_{p}}$, whose exponent is $p^{\lceil(n-m+1) /(p-1)\rceil} \leq$ $p^{\lceil n / 2(p-1)\rceil} \approx \sqrt{\exp G}$.

\section{Higher COCLASS}

In this final section, we investigate Bogomolov and Schur multipliers of finite $p$-groups of a fixed coclass $r$. Referring to [21, 9] we recall a few fundamental facts about coclass theory. Groups of coclass $r$ can be collected into a graph $\Gamma(p, r)$ whose vertices correspond to their isomorphism types and there is an edge between $G$ and $H$ if and only if $G \cong H / N$ where $N$ is the last term of the lower central series of $H$. An infinite pro- $p$ group $S$ of coclass $r$ corresponds precisely to an infinite path in the above graph, and there are only finitely many of them. One can find a minimal positive integer $i$, the so-called primary root (see [9, Section 2.1]), with the property that the coclass of $S / \gamma_{i}(S)$ is precisely $r$ and that the quotient $S / \gamma_{i}(S)$ is not isomorphic to any $\bar{S} / \gamma_{i}(\bar{S})$ for any other pro-p group $\bar{S}$ of coclass $r$. We denote by $\mathcal{T}(S)$ the subtree of $\Gamma(p, r)$ rooted at $S / \gamma_{i}(S)$. The tree $\mathcal{T}(S)$ has a main line given by the lower central quotients of $S$ and it is in general of unbounded width.

As shown by the examples in the previous section, it is possible to find groups with Bogomolov multipliers of arbitrary exponents as one moves further away from the main line. Via the theory of CP covers [17], one can modify these examples to produce groups of arbitrary coclass with the same property. These groups further possess the property that they are stem, i.e., their center is contained in their derived subgroup.

Theorem 4.1. Let $p \geq 5$ be a prime and $r$ a positive integer. For every $x \geq r \geq 1$ there exist stem p-groups of coclass $r$ with Bogomolov multiplier of exponent $p^{x}$.

Proof. Let $H$ be a $p$-group of maximal class with the properties that $\exp \mathrm{B}_{0}(H)=$ $p^{x}$ and that there exists $L \leq \mathrm{B}_{0}(H)$ with $\exp L=p^{x}$ and $\left|\mathrm{B}_{0}(H): L\right|=p^{r-1}$. Such a group $H$ exists by Theorem 1.3. Let $G$ be a CP covering group (see [17, Section 4]) of $H$, and let $Q=G / L$. By [17, Lemma 4.6], the group $G$ is a CP covering group of $Q$ with kernel $L$, and we have $\mathrm{B}_{0}(Q) \cong L$. Therefore $|Q|=|G| /|L|=$ $|H| \cdot\left|\mathrm{B}_{0}(H)\right| /|L|=|H| \cdot p^{r-1}$. At the same time, it follows from [17, Lemma 4.8] that $Q / Z(Q) \cong H / Z(H)$, so $Q$ has the same nilpotency class as $H$. Therefore $Q$ is of coclass $r$. Now, as $H$ is a group of maximal class, its center is equal to the last term of its lower central series, so $H$ is a stem group. The center $Z(Q)$ is generated by $\mathrm{B}_{0}(H) / L$ together with the preimage of $Z(H)$ under the projection $Q \rightarrow H$. Since we also have $\mathrm{B}_{0}(H) / L \leq[Q, Q]$, it follows that $Q$ is a stem group.

In the remainder, we will focus on bounding the sizes of Bogomolov multipliers of groups of fixed coclass. First of all, we recall that the rank of Schur multipliers (and therefore also Bogomolov multipliers) can be absolutely bounded. 
Theorem 4.2 ([1, Theorem 1]). There is a constant $C=C(p, r)$ such that for every finite p-group $G$ of coclass $r$, we have $\mathrm{d}\left(\mathrm{B}_{0}(G)\right) \leq \mathrm{d}(\mathrm{M}(G)) \leq C$.

Bounding the exponent of both multipliers is a more delicate issue. If we only consider finite $p$-groups of fixed coclass that are quotients of an infinite pro- $p$ group, then the exponents of the Schur multipliers are in general unbounded (see [8, Theorem A]). The exponent can nevertheless be bounded in terms of the exponent of the underlying group. This is in accordance with a classical problem asking what the relationship is between the exponent of a finite group and of its Schur multiplier. More precisely, it is conjectured that $\exp \mathrm{M}(G)$ divides $\exp G$ for a finite $p$-group $G$ if $p>2$. Confer [27] and the references therein. In the context of $p$-groups of fixed coclass, it is known that one at least has a linear bound on the exponent of the Schur multiplier.

Theorem 4.3 ([27, Theorem 3.3]). There is a constant $D=D(p, r)$ such that for every finite p-group $G$ of coclass $r$, we have $\exp \mathrm{M}(G) \leq D \cdot \exp G$.

For any group of maximal class, the exponent of the Schur multiplier is bounded by the exponent of the group (see [22, Theorem 1.4]).

As for the Bogomolov multiplier, we now show that there is an absolute bound for the exponent of Bogomolov multipliers of quotients of infinite pro- $p$ groups that are on the main line of the coclass tree.

Theorem 4.4. There is a constant $E=E(p, r)$ such that for every infinite pro-pgroup $S$ of coclass $r$, we have $\exp \mathrm{B}_{0}\left(S / \gamma_{i}(S)\right) \leq E$ for all $i \geq 1$.

Proof. By [7, Theorem 10.1], we have that the group $S$ contains an abelian subgroup $A$ of index bounded in terms of $p$ and $r$. It now follows from [17, Proposition 6.2] that $\exp \mathrm{B}_{0}\left(S / \gamma_{i}(S)\right) \leq\left|S: A \gamma_{i}(S)\right| \cdot \exp \mathrm{B}_{0}\left(A \gamma_{i}(S) / \gamma_{i}(S)\right)=\left|S: A \gamma_{i}(S)\right| \leq \mid S:$ $A \mid$.

The groups in Theorem 1.3 show how groups far away from the main line of the coclass tree $\Gamma(p, 1)$ can have Bogomolov multipliers whose exponents grow without limits. More can be said about the asymptotic structure of both multipliers of groups that are boundedly away from the main line in $\mathcal{T}(S)$ for any pro- $p$ group $S$ of coclass $r$. More precisely, one can consider the shaved subtree $\mathcal{T}(S, k)$ consisting of those groups in $\mathcal{T}(S)$ that are of distance at most $k$ from the main line. Its branches $\mathcal{B}_{j}(S, k)$ consist of descendants of $S / \gamma_{j}(S)$ that are not descendants of $S / \gamma_{j+1}(S)$. It is proved in [10] that these branches satisfy a periodic pattern. This periodicity can be made explicit on the level of groups; each group in the first branch determines an infinite coclass sequence $\left(G_{x}\right)_{x \in \mathbb{N}}$. For each infinite sequence, the groups are uniformly described by a single parameterised presentation (see [9] as well as [11, Chapter 8] for more details), and so their Schur multipliers relate to the parameter $x$ and can also be uniformly described. It is known that sizes of these Schur multipliers will grow as the orders of the underlying groups 
grow (see [8, Theorem A]). Based on the above given bounds for the exponent, the uniform description of Schur multipliers from [9] can be refined and extended to the Bogomolov multiplier as follows.

Theorem 4.5. Let $\left(G_{x}\right)_{x \in \mathbb{N}}$ be an infinite coclass sequence. Then there exist a finite abelian p-group $A$ and integers $s_{1}, \ldots, s_{m}$ such that

$$
\mathrm{M}\left(G_{x}\right) \cong A \times \prod_{i=1}^{m} C_{p^{x+s_{i}}}
$$

for almost all $x$, and for $p>2$ one has $m>0$. Moreover, the groups $\mathrm{B}_{0}\left(G_{x}\right)$ are almost all pairwise isomorphic.

Proof. By [9, Theorem 1] there exist integers $r_{1}, \ldots, r_{m}$ and $s_{1}, \ldots, s_{m}$ so that the Schur multiplier $\mathrm{M}\left(G_{x}\right)$ is of the form

$$
\prod_{i=1}^{m} C_{p^{r_{i} x+s_{i}}}
$$

for almost all $x$. At the same time, it follows from [10, Section 9] (see also [11, Remark 5.23]) that each group $G_{x}$ fits into an exact sequence

$$
1 \longrightarrow T_{x} \longrightarrow G_{x} \longrightarrow R \longrightarrow 1,
$$

where $R$ is a fixed finite $p$-group and $T_{x}$ is a homocyclic abelian group (in fact, an $R$ module) of exponent $p^{x+e}$ for some fixed $e$. This gives that $\exp G_{x} \leq \exp R \cdot \exp T_{x}=$ $\exp R \cdot p^{x+e}$. Now, it follows from Theorem 4.3 that $\exp \mathrm{M}\left(G_{x}\right)$ is linearly bounded in terms of $\exp G_{x}$, which implies that all $r_{1}, \ldots, r_{m}$ must be either 0 or 1 . Together with $[8$, Theorem A], this proves the first claim.

We now turn to Bogomolov multipliers. Suppose $R$ has a polycyclic presentation with $d$ generators $g_{1}, \ldots, g_{d}$, and let $T=\mathbb{Z}_{p}^{n}$, so that $T_{x}=T / p^{x+e} T$. The group $G_{x}$ has a central extension $G_{x}^{*}$, constructed by adding a new central generator $y_{i, j}$ for $1 \leq j \leq i \leq d+n$ as a tail of every one relation in the polycyclic presentation for $G_{x}$ (see [11, Section 8.1]). Suppose there are $\ell$ such relations. Setting $Y_{x}^{*}=\left\langle y_{i, j}\right|$ $1 \leq j \leq i \leq d+n\rangle$, one has $\mathrm{M}\left(G_{x}\right) \cong \operatorname{Torsion}\left(Y_{x}^{*}\right)$. The presentation of $G_{x}^{*}$ might not be consistent (see [11, Section 8.3]), so one needs to impose relations between the central generators $y_{i, j}$ arising from consistency relations. These relations are all of the form

$$
\prod_{1 \leq j \leq i \leq d+n} y_{i, j}^{\theta_{i, j}(x)}=1
$$

for some $\theta_{i, j}(x) \in \mathbb{Z}_{p}\left[p^{x}\right]$, and can therefore be collected into a consistency matrix $A(x)$ with coefficients in $\mathbb{Z}_{p}\left[p^{x}\right]$ (see $[11$, Definition 8.11]). After imposing the consistency relations, one sees that Torsion $\left(Y_{x}^{*}\right)$ is of the form (4.1) (see [11, Theorem 8.14]). Now, it follows from [15, Proposition 2.1] that in order to obtain $\mathrm{B}_{0}\left(G_{x}\right)$, we need to add extra relations to the consistency matrix $A(x)$ in order to obtain a matrix $B(x)$. These relations arise in the following way. For any pair of commuting elements $z_{1}, z_{2}$ in $G_{x}$, we pick their lifts $z_{1}^{*}, z_{2}^{*}$ in the group $G_{x}^{*}$ and add the relation $\left[z_{1}^{*}, z_{2}^{*}\right] \in Y_{x}^{*}$ to the matrix $B(x)$. If $Z_{x}^{*}$ is the group obtained by factoring 
$\mathbb{Z}_{p}^{\ell}$ by the relations imposed by $B(x)$, then we have $\mathrm{B}_{0}\left(G_{x}\right) \cong \operatorname{Torsion}\left(Z_{x}^{*}\right)$. By applying to the same reasoning as in the case of the Schur multiplier, it therefore suffices to show that the relations arising from commuting pairs in $G_{x}$ are of the form (4.3). This can be seen as follows. Fix sections of the projections $G_{x} \rightarrow R$ and $T \rightarrow T_{x}$. Every commuting pair in $G_{x}$ is of the form $w_{1} t_{1}, w_{2} t_{2}$ for $w_{1}, w_{2}$ a lift of a commuting pair in $R$ and $t_{1}, t_{2}$ elements of $T_{x}$.

Suppose first that $w_{1}=w_{2}=1$. This means that we are dealing with commuting pairs in $T_{x}$. For each pair of generators of $T_{x}$, we evaluate their commutator in $G_{x}^{*}$ and obtain $\left[t_{1}, t_{2}\right] \in Y_{x}^{*}$, which is simply one of the generators $y_{i, j}$ and therefore of the form (4.3).

Next, consider the case when $w_{2}=1$. This means we are dealing with commuting pairs of the form $w_{1} t_{1}, t_{2}$. Since we already imposed the relation that $t_{1}, t_{2}$ commute in $Z_{x}^{*}$, it suffices to deal with the case when the commuting pair is of the form $w_{1}, t_{2}$. When $w_{1}$ is fixed, this amounts to specifying the kernel of the associated $\mathbb{Z}_{p}$-linear map $T_{x} \rightarrow T_{x}, t \mapsto\left(1-w_{1}\right) \cdot t$, where $w_{1} \in R$ acts on $T_{x}$ via (4.2). This action is in fact descended from a fixed action of $R$ on $T$ (see [11, Remark 5.23]). Consider then the linear map $1-w_{1}: T \rightarrow T$ and put it into its Smith normal form $X D Y$ with $D=\operatorname{diag}\left(p^{a_{1}}, \ldots, p^{a_{k}}, 0, \ldots, 0\right)$ for some non-negative integers $a_{1} \geq \cdots \geq a_{k}$. Set $v_{i}=Y^{-1} e_{i}$ for $1 \leq i \leq n$. Then the kernel of the induced map $1-w_{1}$ on $T_{x}$ is generated by the vectors

$$
p^{x+e-a_{1}} v_{1}, \ldots, p^{x+e-a_{k}} v_{k}, v_{k+1}, \ldots, v_{n} .
$$

For each one of these generating vectors $v$, we evaluate its commutator with $w_{1}$ in $G_{x}^{*}$ and obtain $\left[w_{1}, v\right] \in Y_{x}^{*}$, which is (by [11, Lemma 8.8]) again of the form (4.3). An analogous argument works when we assume that $w_{1}=1$.

Finally, consider the general case. Let $w_{1}, w_{2}$ be a commuting pair in $R$. In order to determine commuting pairs $w_{1} t_{1}, w_{2} t_{2}$ in $G_{x}$, rewrite the commuting condition as $w_{1} t_{1} w_{2} t_{2}=w_{2} t_{2} w_{1} t_{1}$, which is equivalent to $t_{1}^{w_{2}} t_{2}=\left[w_{2}, w_{1}\right] t_{2}^{w_{1}} t_{1}$. Writing $\left[w_{2}, w_{1}\right]=t^{\alpha(x)}$ for some $t \in T_{x}$ and $\alpha(x) \in \mathbb{Z}_{p}\left[p^{x}\right]$ and interpreting the action of $R$ on $T_{x}$ as a linear map, we can rewrite the commuting condition as

$$
\left(1-w_{2}\right) \cdot t_{1}-\left(1-w_{1}\right) \cdot t_{2}=t^{\alpha(x)} .
$$

This is a linear equation in $t_{1}, t_{2}$. As in the previous case, one can see this as a linear transformation on $T$ and pass to a Smith normal form. We thus get a diagonal equation of the form $D\left[\tilde{t}_{1}, \tilde{t}_{2}\right]^{T}=\left[\tilde{t}^{\tilde{\alpha}(x)}\right]$ for $\tilde{t}_{1}, \tilde{t}_{2}, \tilde{t} \in T$ and $\tilde{\alpha}(x) \in \mathbb{Z}_{p}\left[p^{x}\right]$. The solutions of this equation, if they exist (depending on the initial pair $w_{1}, w_{2}$ ), can be obtained as sums of a particular solution $p_{1}, p_{2}$ and an element $k_{1}, k_{2}$ of the kernel, where both the particular solution and generators of the kernel are of the form $s^{\beta(x)}$ for some $s \in T, \beta(x) \in \mathbb{Z}_{p}\left[p^{x}\right]$ by the same argument as in the previous case. Let us add to the matrix $B(x)$ the relations arising from evaluating in $G_{x}^{*}$ the commutators $\left[w_{1} p_{1} b_{1}, w_{2} p_{2} b_{2}\right],\left[w_{1} p_{1}, w_{2} p_{2}\right] \in Y_{x}^{*}$ for $b_{1}, b_{2}$ basis vectors of the kernel corresponding to the linear equation (4.4). By imposing these relations, we can verify that in the group $G_{x}^{*}$, all commuting pairs of $G_{x}$ lift to commuting pairs. 
Indeed, for $w_{1}, w_{2} \in G_{x}$, particular solutions $p_{1}, p_{2} \in T_{x}$, and elements $k_{1}, k_{2} \in T_{x}$ in the kernel, we can expand the commutator $\left[w_{1} p_{1} k_{1}, w_{2} p_{2} k_{2}\right]$ in $G_{x}^{*}$ modulo the relations collected in $B(x)$ into

$$
\left[w_{1} p_{1}, w_{2} p_{2} k_{2}\right]^{k_{1}}\left[k_{1}, w_{2} p_{2} k_{2}\right]=\left[w_{1} p_{1}, k_{2}\right]\left[w_{1} p_{1}, w_{2} p_{2}\right]\left[k_{1}, w_{2} p_{2}\right] .
$$

Since $p_{1}, p_{2}$ is a particular solution, this further simplifies to $\left[w_{1}, k_{2}\right]\left[k_{1}, w_{2}\right]$. The latter is linear in $k_{1}, k_{2}$, and these can be expanded by the basis of the kernel of (4.4). As all of the commuting relations $\left[w_{1} p_{1} b_{1}, w_{2} p_{2} b_{2}\right]$ have been added to $B(x)$, it follows that $\left[w_{1}, k_{2}\right]\left[k_{1}, w_{2}\right]=1$. We have therefore collected all relations in $Y_{x}^{*}$ induced by commuting pairs in the matrix $B(x)$, and they are all of the form (4.3), as required.

It follows that $\mathrm{B}_{0}\left(G_{x}\right)$ is of the form (4.1) for large enough values of $x$. Now, recall that $G_{x}$ is boundedly far in the graph $\mathcal{T}(S)$ from a group on the mainline. This means that there is a surjection $\pi: G_{x} \rightarrow S_{j}$ for a group $S_{j}=S / \gamma_{j}(S)$ on the main line with ker $\pi$ generated by commutators and $|\operatorname{ker} \pi| \leq p^{C}$ for some constant $C$. Let $z \in \operatorname{ker} \pi \cap Z\left(G_{x}\right)$ be a central commutator of order $p$. It follows from [22, Proposition 4.1] (see also the proof of [17, Theorem 7.6]) that

$$
\operatorname{ker}\left(\mathrm{B}_{0}\left(G_{x}\right) \rightarrow \mathrm{B}_{0}\left(G_{x} /\langle z\rangle\right)\right) \leq J_{z}=\left\langle x \curlywedge y \in G_{x} \curlywedge G_{x} \mid[x, y]=z\right\rangle .
$$

Note that $\exp J_{z}=p$, and therefore $\exp \mathrm{B}_{0}\left(G_{x}\right) \leq p \cdot \exp \mathrm{B}_{0}\left(G_{x} /\langle z\rangle\right)$. Repeating this argument with $G_{x}$ replaced by $G_{x} /\langle z\rangle$, it follows that

$$
\exp \mathrm{B}_{0}\left(G_{x}\right) \leq|\operatorname{ker} \pi| \cdot \exp \mathrm{B}_{0}\left(S_{j}\right) \leq p^{C} \cdot \exp \mathrm{B}_{0}\left(S_{j}\right)
$$

Groups on the main line have bounded Bogomolov multipliers by Theorem 4.4, which means that $\exp \mathrm{B}_{0}\left(G_{x}\right)$ is also bounded. It now follows from the form (4.1) that $\mathrm{B}_{0}\left(G_{x}\right)$ eventually stabilizes for large enough $x$. This completes the proof.

We provide a non-trivial example obtained using GAP [6].

Example 4.6. Consider the following pro-3 group of coclass 3. Let $P$ be the semidirect product of $C_{9}=\langle x\rangle$ by $C_{3}=\langle y\rangle$ with $y$ acting nontrivially on $x$. Let $T=\mathbb{Z}_{3}^{6}=\left\langle t_{1}, \ldots, t_{6}\right\rangle$ be a free 3 -adic abelian group. Let the generators $x, y$ act on $T$ via the matrices

$$
X=\left[\begin{array}{cccccc}
0 & 2 & 1 & -1 & 2 & -2 \\
0 & 1 & 0 & 0 & 1 & -1 \\
0 & 0 & 0 & 0 & 1 & -1 \\
0 & 0 & 0 & 0 & 3 & -2 \\
1 & 1 & 0 & -1 & 0 & -1 \\
0 & 3 & 0 & -1 & 0 & -1
\end{array}\right] \text { and } Y=\left[\begin{array}{cccccc}
1 & 0 & 2 & -2 & -2 & 0 \\
0 & 1 & 1 & -1 & -1 & 0 \\
0 & 0 & 1 & -1 & 0 & 0 \\
0 & 0 & 3 & -2 & 0 & 0 \\
0 & 0 & 0 & 0 & -2 & 1 \\
0 & 0 & 0 & 0 & -3 & 1
\end{array}\right]
$$

The group $S=P \ltimes T$ is a pro-3 group of coclass 3. It has a finite quotient $G=S / \gamma_{5}(S)$ of order $3^{7}$, which is an extension of $\gamma_{3}(S) / \gamma_{5}(S) \cong C_{3} \times C_{3}$ by $P \ltimes T / \gamma_{3}(S)$ with $T / \gamma_{3}(S) \cong C_{3} \times C_{3}$. The Bogomolov multiplier of $G$ is nontrivial, in fact $\mathrm{B}_{0}(G) \cong C_{3}$. Hence all the finite quotients $S / \gamma_{i}(S)$ for $i \geq 5$ have non-trivial Bogomolov multipliers. 


\section{REFERENCES}

[1] H. Abdolzadeh and B. Eick, On efficient presentations for infinite sequences of 2-groups with fixed coclass, Algebra Colloq. 20 (2013), 561-572.

[2] N. Blackburn, On a special class of p-groups, Acta Math. 100 (1958), 45-92.

[3] F. A. Bogomolov, The Brauer group of quotient spaces by linear group actions, Izv. Akad. Nauk SSSR Ser. Mat. 51 (1987), no. 3, 485-516.

[4] H. Chu, S.J. Hu, M. Kang, and B.E. Kunyavskii, Noether's problem and the unramified Brauer group for groups of order 64, Int. Math. Res. Not. 12 (2010), 2329-2366.

[5] H. Chu, S.J. Hu, M. Kang, and Y.G. Prokhorov, Noether's problem for groups of order 32, J. Algebra 320 (2008), 3022-3035.

[6] H. Dietrich, B. Eick, and D. Feichtenschlager, Investigating p-groups by coclass with GAP, In Computational group theory and the theory of groups, Contemp. Math. 470, 45-61, AMS, Providence, RI, 2008.

[7] J.D. Dixon M.P.F. du Sautoy, A. Mann, D. Segal, Analytic Pro-p-Groups, Cambridge University Press London Math. Soc. Lecture Note Series 157, 1991.

[8] B. Eick, Schur multiplicators of infinite pro-p-groups with finite coclass, Israel J. Math 166 1 (2008), 147-156.

[9] B. Eick and D. Feichtenschlager, Computation of low-dimensional (co)homology groups for infinite sequences of p-groups with fixed coclass, International Journal of Algebra and Computation 214 (2011), 635-649.

[10] B. Eick and C. Leedham-Green, On the classification of prime-power groups by coclass, Bull. Lond. Math. Soc., 40 (2008), 274-288.

[11] D. Feichtenschlager, Symbolic computation with infinite sequences of p-groups with fixed coclass, Logos Verlag Berlin GmbH, 2010.

[12] G. A. Fernández-Alcober, The exact lower bound for the degree of commutativity of a p-group of maximal class, J. Algebra 174 (1995), 523-530.

[13] G. A. Fernández-Alcober, An introduction to finite p-groups: regular p-groups and groups of maximal class, Mat. Contemp. 20 (2001), 155-226.

[14] B. Huppert, Endliche Gruppen, I, Springer, Berlin, 1967.

[15] U. Jezernik and P. Moravec, Bogomolov multipliers of groups of order 128, Exp. Math. 23 (2014), no. 2, 174-180.

[16] U. Jezernik and P. Moravec, Universal commutator relations, Bogomolov multipliers, and commuting probability, J. Algebra 428 (2015), 1-25.

[17] U. Jezernik and P. Moravec, Commutativity preserving extensions of groups, Proc. Roy. Soc. Edinburgh Sect. A 148 (2018), 575-592.

[18] C.R. Leedham-Green and S. McKay, On p-groups of maximal class, I, Quart. J. Math. 27 (3) (1976), 297-311.

[19] C.R. Leedham-Green and S. McKay, On p-groups of maximal class, II, Quart. J. Math. 29 (2) (1978), 175-186.

[20] C.R. Leedham-Green and S. McKay, On p-groups of maximal class, III, Quart. J. Math. 29 (3) (1978), 281-299.

[21] C.R. Leedham-Green and S. McKay, The structure of groups of prime power order, Vol. 27, Oxford University Press, Oxford, 2002.

[22] P. Moravec, On the Schur multipliers of finite p-groups of given coclass, Israel J. Math $\mathbf{1 8 5}$ 1 (2011), 189-205.

[23] P. Moravec, Groups of order $p^{5}$ and their unramified Brauer groups, J. Algebra 372 (2012), 320-327.

[24] P. Moravec, Unramified Brauer groups finite and infinite groups, Amer. J. Math. 134 (2012), no. $6,1679-1704$

[25] E. Noether, Rationale Funktionenkörper, Jahrbericht Deutsch. Math.-Verein. 22 (1913), 316319 .

[26] D.J. Saltman, Noether's problem over an algebraically closed field, Invent. Math. 77 (1984), $71-84$.

[27] N. Sambonet, Bounds for the exponent of the Schur multiplier, Journal of Pure and Applied Algebra 221 (2017), 2053-2063.

[28] I. Schur, Über die Darstellung der endlichen Gruppen durch gebrochene lineare Substitutionen, J. Reine Angew. Math. 127 (1904), 20-50.

[29] R. Shepherd, p-Groups of maximal class, PhD thesis, University of Chicago, 1970. 
(Gustavo A. Fernández-Alcober) Department of Mathematics, University of the Basque Country UPV/EHU, 48080 Bilbao, Spain

E-mail address: gustavo.fernandez@ehu.eus

(Urban Jezernik) Department of Mathematics, University of the Basque Country UPV/EHU, 48080 BILBAO, SPAIN

E-mail address: urban.jezernik@ehu.eus 